\title{
Wireless Detection, Identification and Reading of Passive Electromagnetic Sensors based on Beam-Steering FMCW RADAR
}

\author{
H. Aubert* ${ }^{(1,2)}$, P. Pons ${ }^{(1)}$ and D. Henry ${ }^{(1,2)}$
}

(1) CNRS, LAAS, 7 avenue du colonel Roche, F-31400 Toulouse, France, http://www.laas.fr (2) Univ. of Toulouse, INPT, LAAS, F-31400 Toulouse, France, http://www.inp-toulouse.fr

The wireless measurement of a physical quantity from the analysis of the RADAR Cross Section (RCS) variability of passive (battery-less) sensors was proposed for the first time by the authors and their collaborators in 2008 while the proof-of-concept was demonstrated in 2010. A millimeter-wave (mm-wave) Frequency-Modulated Continuous-Wave (FMCW) RADAR was used for the measurement of RCS variation and for the remote derivation of the physical quantity changes. The choice of mm-wave frequency range facilitates the design of multi-beam or beam-steering FMCW RADAR for interrogating sensors simultaneously or sequentially in many directions in space. The wireless sensing technique based on RCSvariability measurement has been successfully applied to the remote estimation of many physical quantities, such as temperature (2010-2012) and strain (2013). Very recent applications using MEMS technology of the proposed wireless sensing technique based on RCS-variability measurement will be presented at the conference. Moreover the wireless detection, identification and reading of such sensors from the analysis of 3D RADAR images will be discussed. These images were obtained by using a beam-steering FMCW RADAR. For illustration purpose Figure 1 shows RADAR images of a scanned region containing passive sensors obtained from a $24 \mathrm{GHz}$ FMCW RADAR (bandwidth: $2 \mathrm{GHz}$ ). The RADAR used mechanical beam-steering in elevation and in azimuth. The monitoring of the transmitting RADAR antenna was performed by computer. Such automatized 3D RADAR scanning system was advantageously used by the authors for the detection, identification and remote reading of passive electromagnetic sensors. Very recent results will be reported and discussed at the conference. The technical challenges and possible solutions regarding the proposed wireless interrogation of passive EM sensors will be presented.

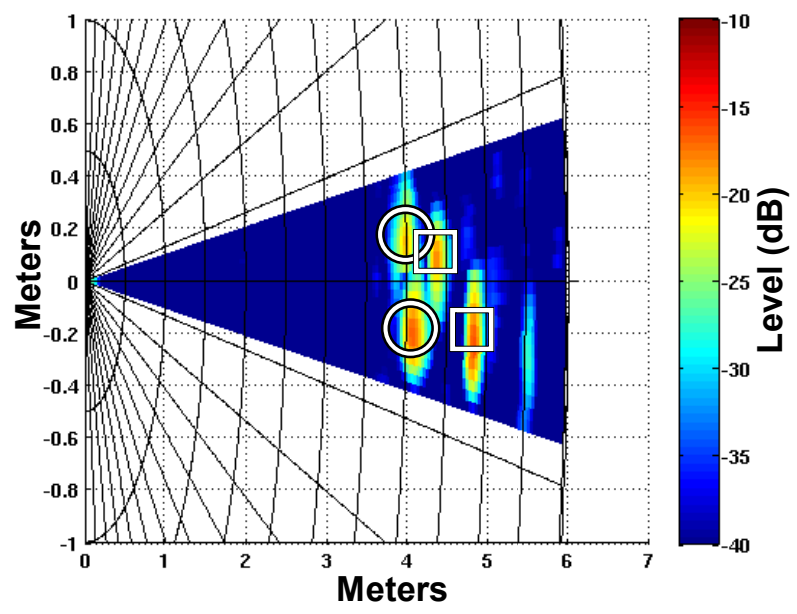

(a)

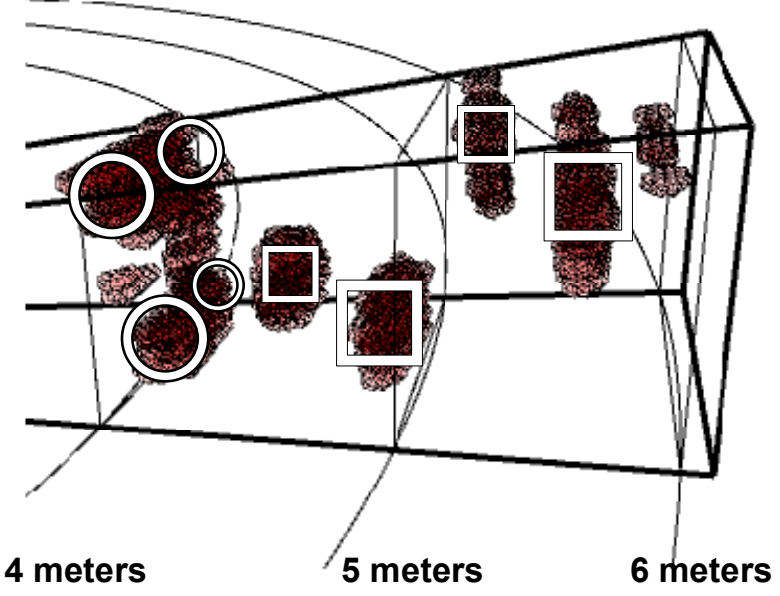

(b)

Figure 1: RADAR images of a scanned region containing passive sensors:

(a) 2D visualization in a given cut-plane and (b) 3D visualization of echo levels higher than a given threshold (measurement results). The circles indicate the EM echo associated with the structural scattering mode of the passive sensors while the squares give the location of the sensing scattering modes. 